\title{
MATRIX Mascot Search Results
}

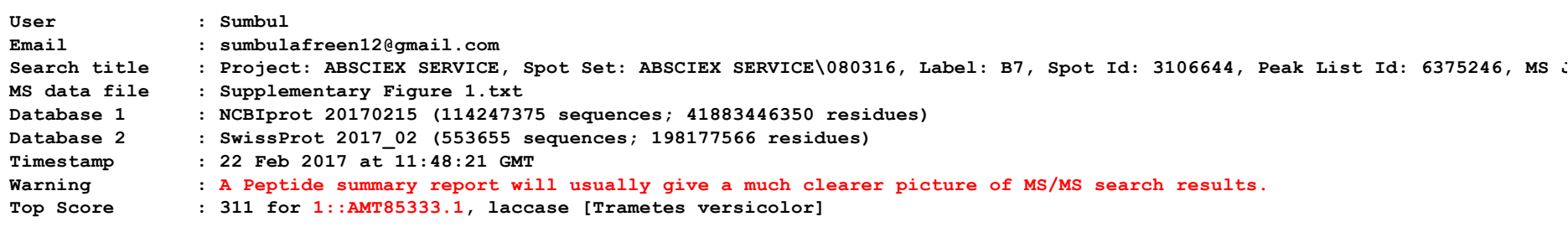

\section{Mascot Score Histogram}

Protein score is $-10 * \log (\mathrm{P})$, where $\mathrm{P}$ is the probability that the observed match is a random event.

Protein scores greater than 93 are significant $(\mathrm{p}<0.05)$.

Protein scores are derived from ions scores as a non-probabilistic basis for ranking protein hits.

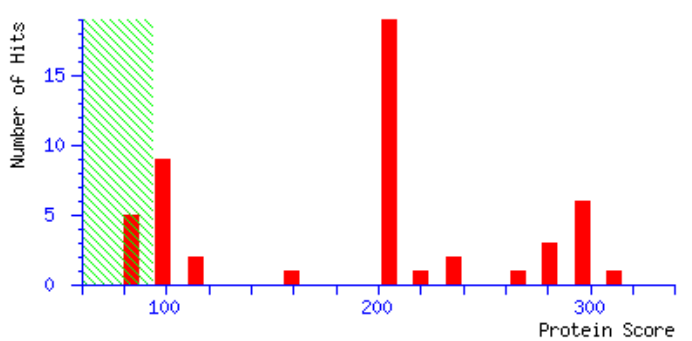

Protein Summary Report

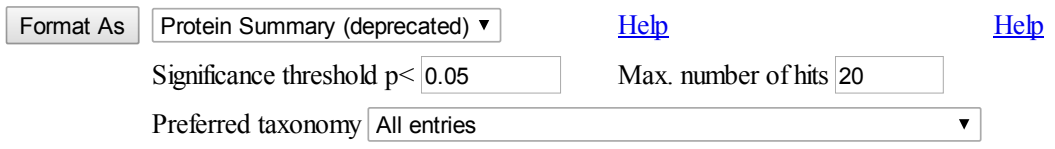

Re-Search All Search Unmatched

Index
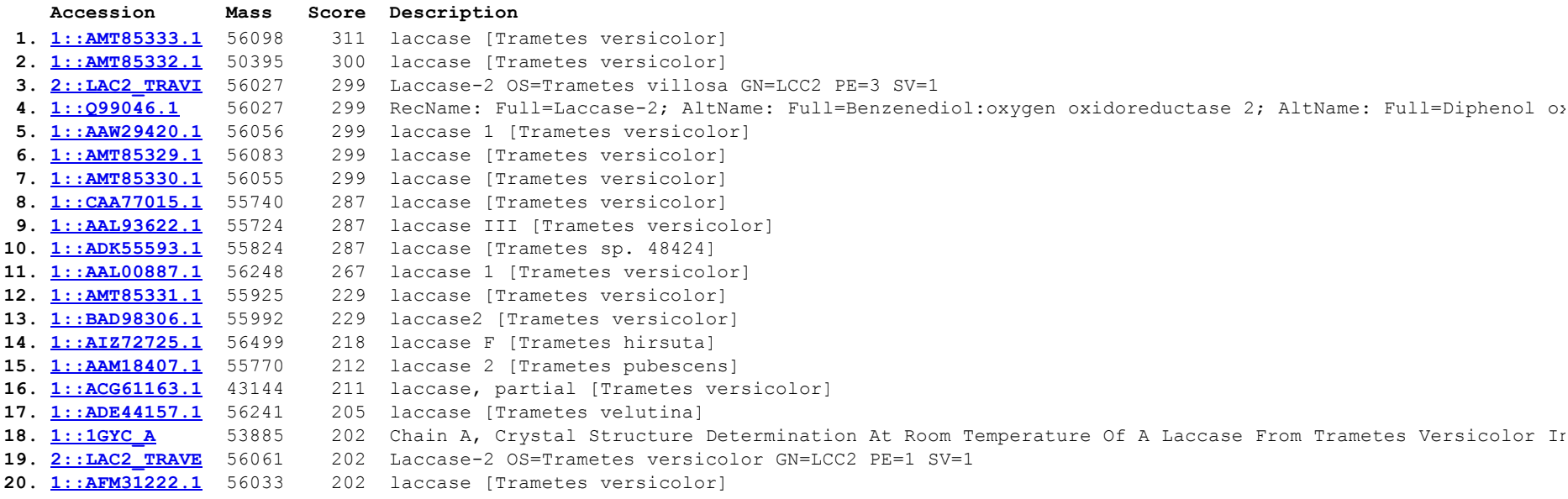

\section{Results List}

laccase [Trametes versicolor]

Observed Mr (expt) Mr (calc)

$1514.7855 \quad 1513.7782 \quad 1513.8253$

$\begin{array}{lll}1975.9897 & 1974.9824 & 1975.0276\end{array}$

$\begin{array}{lll}2470.0762 & 2469.0689 & 2469.1448\end{array}$

$\begin{array}{lll}3214.6296 & 3213.6223 & 3213.7034\end{array}$

(1)

No match to: $731.4565,745.4716,751.3851,761.4732,804.2769,819.5120,832.3038,1243.6450,1474.6757,832.3132,834.3159$,
$1703.7522,1742.7133,1948.0842,1991.9727,2021.9513,2043.0476,2070.0222,2087.9597,2101.9629,2150.1257,2199.0493$,
$1243.6581,1474.6874,1514.7983,1515.7916,3247.3425,1703.7673,1705.7672,1974.0721,1976.0077,1991.9894,2059.9446$,

$1243.6581,1474.6874,1514.7983,1515.7916,3247.3425,1703.7673,1705.7672,1$
$2087.9773,2150.1462,2158.0591,2199.0723,2470.0964,3214.6680,3247.3762$

2

\begin{tabular}{|c|c|c|c|c|c|c|c|c|}
\hline \multicolumn{2}{|c|}{$1::$ AMT 85332.1} & 50395 & \multicolumn{2}{|c|}{ Score: 300} & Expect & \multicolumn{2}{|c|}{$: 1.1 \mathrm{e}-22$} & \multirow[t]{2}{*}{ Matches: 4} \\
\hline lo & rametes & & & & & & & \\
\hline Observed & $M r(\exp t)$ & $\operatorname{Mr}(\operatorname{calc})$ & Delta & Start & End & Miss & Ions & Peptide \\
\hline 1514.7855 & 1513.7782 & 1513.8253 & -0.0470 & 182 & -196 & 0 & 38 & R.FPLGADATLINGLGR.S \\
\hline 1975.9897 & 1974.9824 & 1975.0276 & -0.0451 & 281 & -300 & 0 & 92 & R.ANPNFGTVGFAGGINSAILR.Y \\
\hline 2470.0762 & 2469.0689 & 2469.1448 & -0.0759 & 157 & -177 & 0 & 66 & R.YDVDNESTVITLTDWYHTAAR. L \\
\hline
\end{tabular}


2/22/2017 Protein Summary Report (Project: ABSCIEX SERVICE, Spot Set: ABSCIEX SERVICEI080316, Label: B7, Spot Id: 3106644, Peak List Id: 6375246, MS...

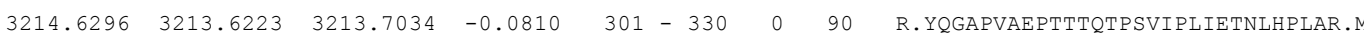

No match to: $731.4565,745.4716,751.3851,761.4732,804.2769,819.5120,832.3038,1243.6450,1474.6757,832.3132,834.3159$, $1703.7522,1742.7133,1948.0842,1991.9727,2021.9513,2043.0476,2070.0222,2087.9597,2101.9629,2150.1257,2199.0493$, $1243.6581,1474.6874,1514.7983,1515.7916,3247.3425,1703.7673,1705.7672,1974.0721,1976.0077,1991.9894,2059.9446$, $2070.0339,2087.9773,2150.1462,2158.0591,2199.0723,2470.0964,3214.6680,3247.3762$

3.

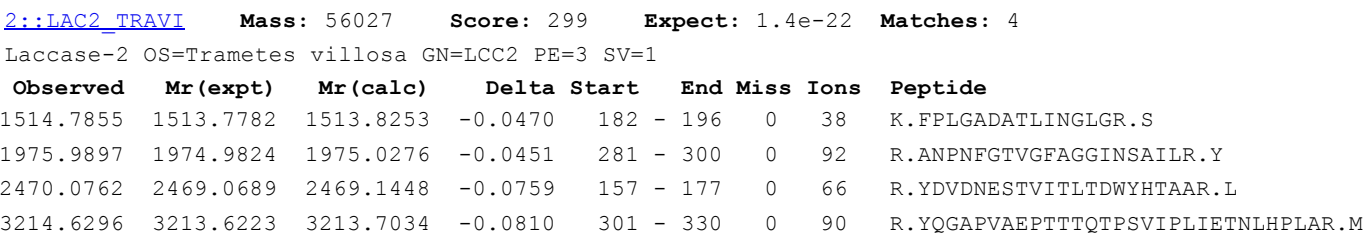

No match to: $731.4565,745.4716,751.3851,761.4732,804.2769,819.5120,832.3038,1243.6450,1474.6757,832.3132,834.3159$, $1703.7522,1742.7133,1948.0842,1991.9727,2021.9513,2043.0476,2070.0222,2087.9597,2101.9629,2150.1257,2199.0493$, $1243.6581,1474.6874,1514.7983,1515.7916,3247.3425,1703.7673,1705.7672,1974.0721,1976.0077,1991.9894,2059.9446$, $2070.0339,2087.9773,2150.1462,2158.0591,2199.0723,2470.0964,3214.6680,3247.3762$

4. 1::099046.1 Mass: 56027 Score: 299 Expect: $1.4 \mathrm{e}-22$ Matches: 4

RecName: Full=Laccase-2; AltName: Full=Benzenediol:oxygen oxidoreductase 2; AltName: Full=Diphenol oxidase 2; AltName: Full=Uri Observed Mr (expt) Mr(calc) Delta Start End Miss Ions Peptide

$\begin{array}{lllllllll}1514.7855 & 1513.7782 & 1513.8253 & -0.0470 & 182 & -196 & 0 & 38 & \text { K.FPLGADATLINGLGR.S }\end{array}$

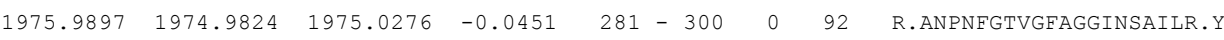

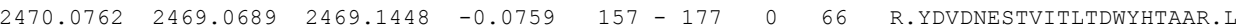

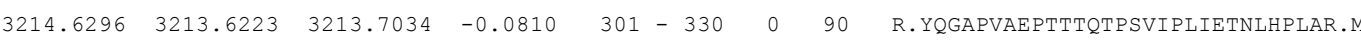

No match to: $731.4565,745.4716,751.3851,761.4732,804.2769,819.5120,832.3038,1243.6450,1474.6757,832.3132,834.3159$, $1703.7522,1742.7133,1948.0842,1991.9727,2021.9513,2043.0476,2070.0222,2087.9597,2101.9629,2150.1257,2199.0493$, $1243.6581,1474.6874,1514.7983,1515.7916,3247.3425,1703.7673,1705.7672,1974.0721,1976.0077,1991.9894,2059.9446$, $2070.0339,2087.9773,2150.1462,2158.0591,2199.0723,2470.0964,3214.6680,3247.3762$

5.

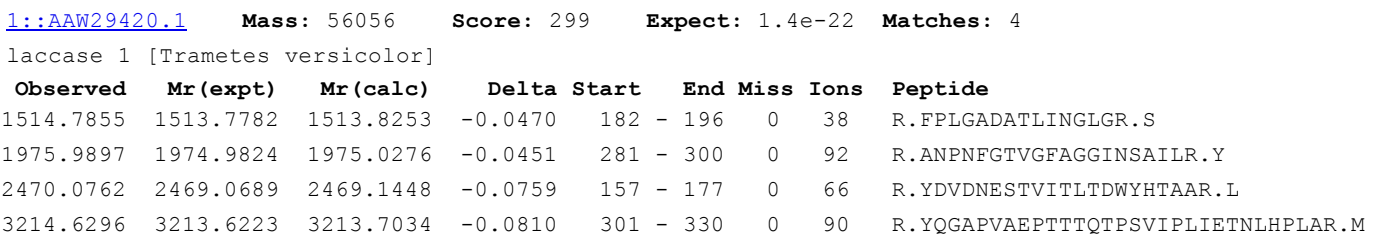

No match to: $731.4565,745.4716,751.3851,761.4732,804.2769,819.5120,832.3038,1243.6450,1474.6757,832.3132,834.3159$, $1703.7522,1742.7133,1948.0842,1991.9727,2021.9513,2043.0476,2070.0222,2087.9597,2101.9629,2150.1257,2199.0493$, $1243.6581,1474.6874,1514.7983,1515.7916,3247.3425,1703.7673,1705.7672,1974.0721,1976.0077,1991.9894,2059.9446$, $2070.0339,2087.9773,2150.1462,2158.0591,2199.0723,2470.0964,3214.6680,3247.3762$

6. 1::AMT85329.1 Mass: 56083

laccase [Trametes versicolor]

Observed Mr (expt) Mr(calc)

$1514.7855 \quad 1513.7782 \quad 1513.8253$

$1975.9897 \quad 1974.9824 \quad 1975.0276$

$\begin{array}{lll}2470.0762 & 2469.0689 & 2469.1448\end{array}$

$3214.6296 \quad 3213.6223 \quad 3213.7034$

Score: 299 Expect: $1.4 \mathrm{e}-22$ Matches: 4 17522, 1742.7133, 1948.0842, 1991.9727, 2021.9513, 2043.0476, 2070.0222, 2087.9597, 2101.9629, 2150.1257, 2199.0493, $1243.6581,1474.6874,1514.7983,1515.7916,3247.3425,1703.7673,1705.7672,1974.0721,1976.0077,1991.9894,2059.9446$, $2070.0339,2087.9773,2150.1462,2158.0591,2199.0723,2470.0964,3214.6680,3247.3762$

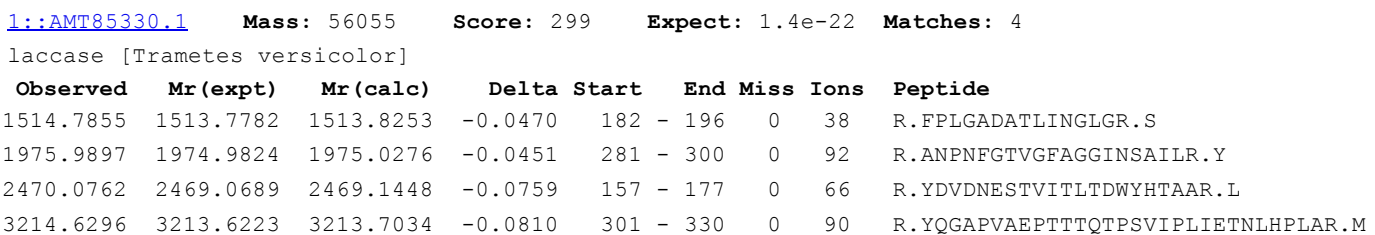

No match to: $731.4565,745.4716,751.3851,761.4732,804.2769,819.5120,832.3038,1243.6450,1474.6757,832.3132,834.3159$, 1703.7522, 1742.7133, 1948.0842, 1991.9727, 2021.9513, 2043.0476, 2070.0222, 2087.9597, 2101.9629, 2150.1257, 2199.0493, $1243.6581,1474.6874,1514.7983,1515.7916,3247.3425,1703.7673,1705.7672,1974.0721,1976.0077,1991.9894,2059.9446$, $2070.0339,2087.9773,2150.1462,2158.0591,2199.0723,2470.0964,3214.6680,3247.3762$

8 .

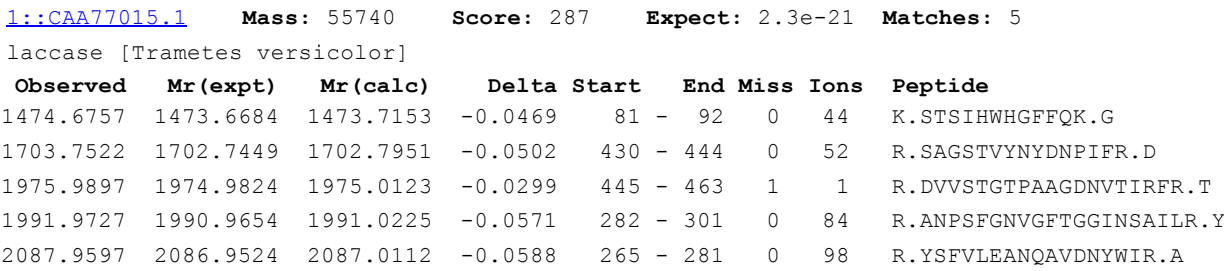

No match to: $731.4565,745.4716,751.3851,761.4732,804.2769,819.5120,832.3038,1243.6450,1514.7855,832.3132,834.3159$, $1742.7133,1948.0842,2021.9513,2043.0476,2070.0222,2101.9629,2150.1257,2199.0493,2470.0762,1243.6581,1474.6874$, $1514.7983,1515.7916,3214.6296,3247.3425,1703.7673,1705.7672,1974.0721,1976.0077,1991.9894,2059.9446,2070.0339$, $2087.9773,2150.1462,2158.0591,2199.0723,2470.0964,3214.6680,3247.3762$

9. 1::AAL93622.1 Mass: 55724 Score: 287 Expect: $2.3 e-21$ Matches: 5

laccase III [Trametes versicolor]

Observed Mr(expt) Mr(calc) Delta Start End Miss Ions Peptide

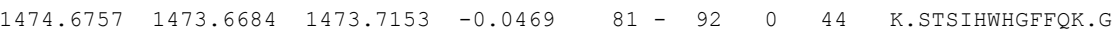

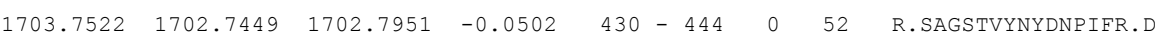


2/22/2017 Protein Summary Report (Project: ABSCIEX SERVICE, Spot Set: ABSCIEX SERVICEI080316, Label: B7, Spot Id: 3106644, Peak List Id: 6375246, MS...

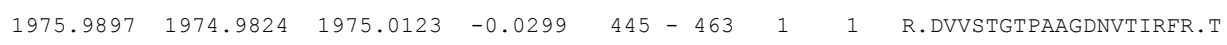

$\begin{array}{lllllllll}1991.9727 & 1990.9654 & 1991.0225 & -0.0571 & 282 & -301 & 0 & 84 & \text { R.ANPSFGNVGFTGGINSAILR.Y }\end{array}$

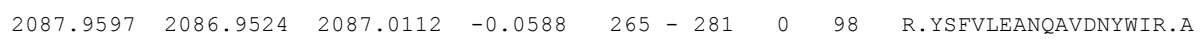

No match to: $731.4565,745.4716,751.3851,761.4732,804.2769,819.5120,832.3038,1243.6450,1514.7855,832.3132,834.3159$, $1742.7133,1948.0842,2021.9513,2043.0476,2070.0222,2101.9629,2150.1257,2199.0493,2470.0762,1243.6581,1474.6874$, $1514.7983,1515.7916,3214.6296,3247.3425,1703.7673,1705.7672,1974.0721,1976.0077,1991.9894,2059.9446,2070.0339$, $2087.9773,2150.1462,2158.0591,2199.0723,2470.0964,3214.6680,3247.3762$

10. $1:$ :ADK55593.1 Mass: 55824

laccase [Trametes sp. 48424]

\section{Observed Mr (expt) Mr(calc) Delta Start End Miss Ions Peptide}

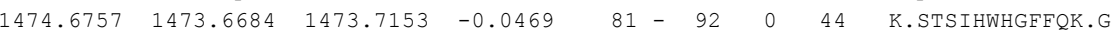

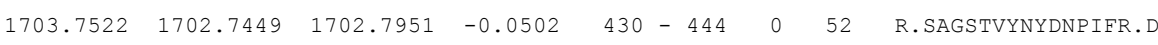

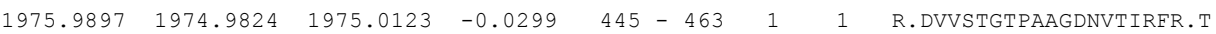

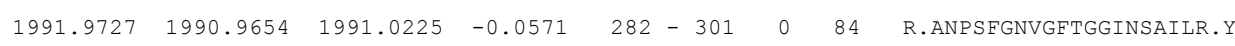

$\begin{array}{llllllll}2087.9597 & 2086.9524 & 2087.0112 & -0.0588 & 265 & -281 & 0 & 98\end{array}$

No match to: $731.4565,745.4716,751.3851,761.4732,804.2769,819.5120,832.3038,1243.6450,1514.7855,832.3132,834.3159$, $1742.7133,1948.0842,2021.9513,2043.0476,2070.0222,2101.9629,2150.1257,2199.0493,2470.0762,1243.6581,1474.6874$, $1514.7983,1515.7916,3214.6296,3247.3425,1703.7673,1705.7672,1974.0721,1976.0077,1991.9894,2059.9446,2070.0339$, $2087.9773,2150.1462,2158.0591,2199.0723,2470.0964,3214.6680,3247.3762$

11.

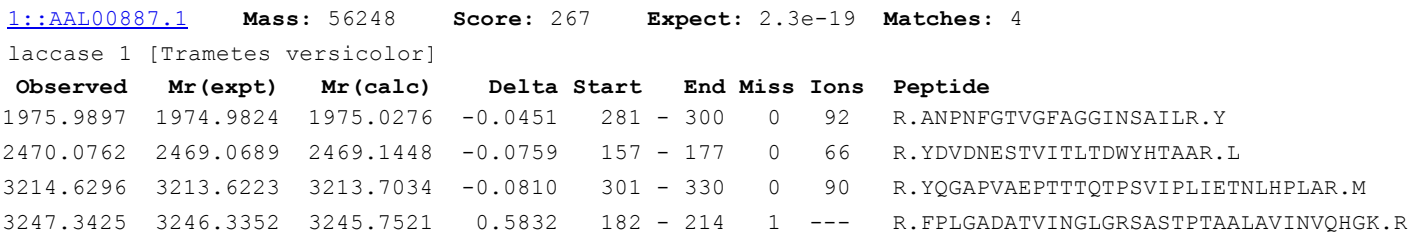

No match to: $731.4565,745.4716,751.3851,761.4732,804.2769,819.5120,832.3038,1243.6450,1474.6757,1514.7855,832.3132$, $834.3159,1703.7522,1742.7133,1948.0842,1991.9727,2021.9513,2043.0476,2070.0222,2087.9597,2101.9629,2150.1257$, $2199.0493,1243.6581,1474.6874,1514.7983,1515.7916,1703.7673,1705.7672,1974.0721,1976.0077,1991.9894,2059.9446$, $2070.0339,2087.9773,2150.1462,2158.0591,2199.0723,2470.0964,3214.6680,3247.3762$

12. 1::AMT85331.1 Mass: 55925 Score: 229 Expect: $1.4 \mathrm{e}-15$ Matches: 3

laccase [Trametes versicolor]

\section{Observed Mr(expt) Mr(calc) Delta Start End Miss Ions Peptide}

$\begin{array}{llllllll}1514.7855 & 1513.7782 & 1513.8253 & -0.0470 & 181-195 & 0 & 38 & \text { R.FPLGADATLINGLGR.S }\end{array}$

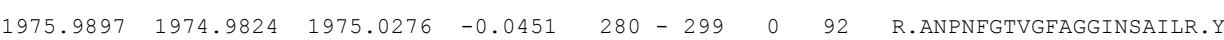

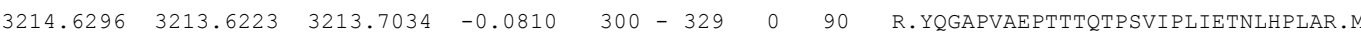

No match to: $731.4565,745.4716,751.3851,761.4732,804.2769,819.5120,832.3038,1243.6450,1474.6757,832.3132,834.3159$, $1703.7522,1742.7133,1948.0842,1991.9727,2021.9513,2043.0476,2070.0222,2087.9597,2101.9629,2150.1257,2199.0493$, $2470.0762,1243.6581,1474.6874,1514.7983,1515.7916,3247.3425,1703.7673,1705.7672,1974.0721,1976.0077,1991.9894$, $2059.9446,2070.0339,2087.9773,2150.1462,2158.0591,2199.0723,2470.0964,3214.6680,3247.3762$

13. 1::BAD98306.1 Mass: 55992

laccase2 [Trametes versicolor]

Observed $\quad \mathrm{Mr}$ (expt) $\quad \mathrm{Mr}(\mathrm{calc})$

$1514.7855 \quad 1513.7782 \quad 1513.8253$

$1975.9897 \quad 1974.9824 \quad 1975.0276$

Score: 229 Expect: $1.4 \mathrm{e}-15$ Matches: 3

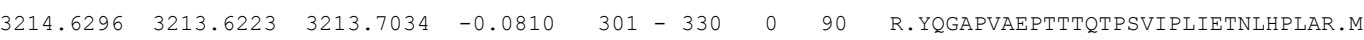

No match to: $731.4565,745.4716,751.3851,761.4732,804.2769,819.5120,832.3038,1243.6450,1474.6757,832.3132,834.3159$, $1703.7522,1742.7133,1948.0842,1991.9727,2021.9513,2043.0476,2070.0222,2087.9597,2101.9629,2150.1257,2199.0493$, $2470.0762,1243.6581,1474.6874,1514.7983,1515.7916,3247.3425,1703.7673,1705.7672,1974.0721,1976.0077,1991.9894$, $2059.9446,2070.0339,2087.9773,2150.1462,2158.0591,2199.0723,2470.0964,3214.6680,3247.3762$

\begin{tabular}{|c|c|c|c|c|c|c|c|c|}
\hline Observed & Mr (expt) & $\operatorname{Mr}(\mathrm{calc})$ & Delta & Start & End & Miss & Ions & Peptide \\
\hline .474 .6757 & 1473.6684 & 1473.7153 & -0.0469 & 85 & 96 & 0 & 44 & K.STS I HWHGFFQK. G \\
\hline 975.9897 & 1974.9824 & 1976.0480 & -1.0655 & 286 & -305 & 0 & 73 & R.ANPLFGTTGEAGGINSAILR.Y \\
\hline 087.9597 & 2086.9524 & 2087.0112 & -0.0588 & 269 & -285 & 0 & 98 & R.YSFVLEANQAVDNYWIR.A \\
\hline
\end{tabular}

$\begin{array}{lllllll}2087.9597 & 2086.9524 & 2087.0112 & -0.0588 & 269 & -285 & 0\end{array}$

No match to: $731.4565,745.4716,751.3851,761.4732,804.2769,819.5120,832.3038,1243.6450,1514.7855,832.3132,834.3159$, 1703.7522, 1742.7133, 1948.0842, 1991.9727, 2021.9513, 2043.0476, 2070.0222, 2101.9629, 2150.1257, 2199.0493, 2470.0762, $1243.6581,1474.6874,1514.7983,1515.7916,3214.6296,3247.3425,1703.7673,1705.7672,1974.0721,1976.0077,1991.9894$, $2059.9446,2070.0339,2087.9773,2150.1462,2158.0591,2199.0723,2470.0964,3214.6680,3247.3762$

15. 1::AAM18407.1 Mass: 55770

laccase 2 [Trametes pubescens]

Observed Mr (expt) Mr(calc)

$\begin{array}{lll}1474.6757 & 1473.6684 & 1473.7153\end{array}$

$\begin{array}{lll}1474.6757 & 1473.6684 & 1473.7153 \\ 1703.7522 & 1702.7449 & 1702.7951\end{array}$

$1975.9897 \quad 1974.9824 \quad 1975.0123$

$2087.9597 \quad 2086.9524 \quad 2087.0112$

$2158.0591 \quad 4314.1036 \quad 4313.2631$

Score: 212 Expect: $7.2 e-14$ Matches: 5

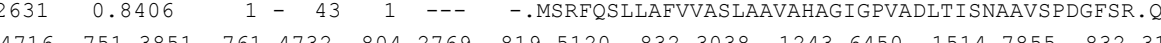

( $751.3851,761.4732,804.2769,819.5120,832.3038,1243.6450,1514.7855,832.3132,834.3159$, $1742.7133,1948.0842,1991.9727,2021.9513,2043.0476,2070.0222,2101.9629,2150.1257,2199.0493,2470.0762,1243.6581$, $1474.6874,1514.7983,1515.7916,3214.6296,3247.3425,1703.7673,1705.7672,1974.0721,1976.0077,1991.9894,2059.9446$, $2070.0339,2087.9773,2150.1462,2199.0723,2470.0964,3214.6680,3247.3762$

16. 1::ACG61163.1 Mass: 43144 Score: 211 Expect: 9.1e-14 Matches: 4

laccase, partial [Trametes versicolor]

Observed Mr(expt) Mr(calc) Delta Start End Miss Ions Peptide

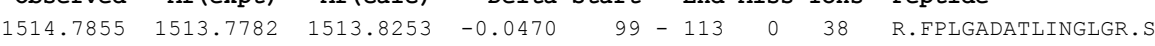

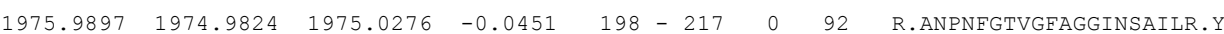


2/22/2017 Protein Summary Report (Project: ABSCIEX SERVICE, Spot Set: ABSCIEX SERVICEl080316, Label: B7, Spot Id: 3106644, Peak List Id: 6375246, MS...

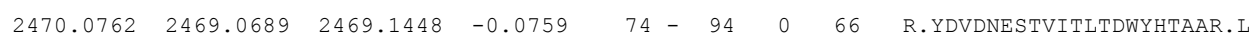
$3247.3425 \quad 3246.3352 \quad 3245.7408 \quad 0.5944 \quad 218-247 \quad 1 \quad--\quad$ R.YRGAPVAEPTTTQTTSVIPLIETNLHPLAR.M No match to: $731.4565,745.4716,751.3851,761.4732,804.2769,819.5120,832.3038,1243.6450,1474.6757,832.3132,834.3159$, 1703.7522, 1742.7133, 1948.0842, 1991.9727, 2021.9513, 2043.0476, 2070.0222, 2087.9597, 2101.9629, 2150.1257, 2199.0493, $1243.6581,1474.6874,1514.7983,1515.7916,3214.6296,1703.7673,1705.7672,1974.0721,1976.0077,1991.9894,2059.9446$, $2070.0339,2087.9773,2150.1462,2158.0591,2199.0723,2470.0964,3214.6680,3247.3762$

17.

\begin{tabular}{|c|c|c|c|c|c|c|c|c|}
\hline bserved & $\operatorname{Mr}($ expt $)$ & $\operatorname{Mr}(\operatorname{calc})$ & Delta & Start & End & Miss & Ions & Peptide \\
\hline 514.7855 & 1513.7782 & 1513.8253 & -0.0470 & 183 & -197 & 0 & 38 & K.FPLGADATLINGLGR.S \\
\hline 70.0762 & 2469.0689 & 2469.1448 & -0.0759 & 158 & -178 & 0 & 66 & R.YDVDNESTVITLTDWYHTAAR.L \\
\hline 14.6296 & 3213.6223 & 3213.7034 & -0.0810 & 302 & -331 & 0 & 90 & R.YQGAPVAEPTTTQTPSVIPLIETNLHPLAR.M \\
\hline
\end{tabular}

No match to: $731.4565,745.4716,751.3851,761.4732,804.2769,819.5120,832.3038,1243.6450,1474.6757,832.3132,834.3159$, $1703.7522,1742.7133,1948.0842,1975.9897,1991.9727,2021.9513,2043.0476,2070.0222,2087.9597,2101.9629,2150.1257$, $2199.0493,1243.6581,1474.6874,1514.7983,1515.7916,3247.3425,1703.7673,1705.7672,1974.0721,1976.0077,1991.9894$, $2059.9446,2070.0339,2087.9773,2150.1462,2158.0591,2199.0723,2470.0964,3214.6680,3247.3762$

18. 1::1GYC_A Mass: 53885 Score: 202 Expect: $7.2 e-13$ Matches: 3

Chain A, Crystal Structure Determination At Room Temperature of A Laccase From Trametes Versicolor In Its Oxidised Form Contain Observed Mr (expt) Mr(calc) Delta Start End Miss Ions Peptide $\begin{array}{lllllllll}1514.7855 & 1513.7782 & 1513.8253 & -0.0470 & 162 & -176 & 0 & 38 & \text { R.FPLGADATLINGLGR.S }\end{array}$

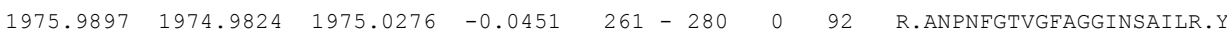
$\begin{array}{lllllllll}2470.0762 & 2469.0689 & 2469.1448 & -0.0759 & 137 & -157 & 0 & 66 & \text { R.YDVDNESTVITLTDWYHTAAR.L }\end{array}$

No match to: $731.4565,745.4716,751.3851,761.4732,804.2769,819.5120,832.3038,1243.6450,1474.6757,832.3132,834.3159$, 1703.7522, 1742.7133, 1948.0842, 1991.9727, 2021.9513, 2043.0476, 2070.0222, 2087.9597, 2101.9629, 2150.1257, 2199.0493, $1243.6581,1474.6874,1514.7983,1515.7916,3214.6296,3247.3425,1703.7673,1705.7672,1974.0721,1976.0077,1991.9894$, $2059.9446,2070.0339,2087.9773,2150.1462,2158.0591,2199.0723,2470.0964,3214.6680,3247.3762$

19. 2::LAC2 TRAVE Mass: 56061 Score: 202 Expect: $7.2 \mathrm{e}-13$ Matches: 3

Laccase-2 OS=Trametes versicolor GN=LCC2 $\mathrm{PE}=1 \mathrm{SV}=1$

Observed $\quad \operatorname{Mr}$ (expt) $\quad \mathrm{Mr}$ (calc) Delta Start End Miss Ions Peptide

$\begin{array}{lllllll}1514.7855 & 1513.7782 & 1513.8253 & -0.0470 & 182 & -196 & 0\end{array}$ R.FPLGADATLINGLGR.S

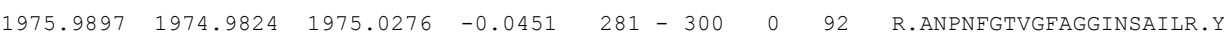

$2470.0762 \quad 2469.06892469 .1448-0.0759 \quad 157-177 \quad 0 \quad 66$ R.YDVDNESTVITLTDWYHTAAR.I

No match to: $731.4565,745.4716,751.3851,761.4732,804.2769,819.5120,832.3038,1243.6450,1474.6757,832.3132,834.3159$, 1703.7522, 1742.7133, 1948.0842, 1991.9727, 2021.9513, 2043.0476, 2070.0222, 2087.9597, 2101.9629, 2150.1257, 2199.0493, $1243.6581,1474.6874,1514.7983,1515.7916,3214.6296,3247.3425,1703.7673,1705.7672,1974.0721,1976.0077,1991.9894$, $2059.9446,2070.0339,2087.9773,2150.1462,2158.0591,2199.0723,2470.0964,3214.6680,3247.3762$

20.

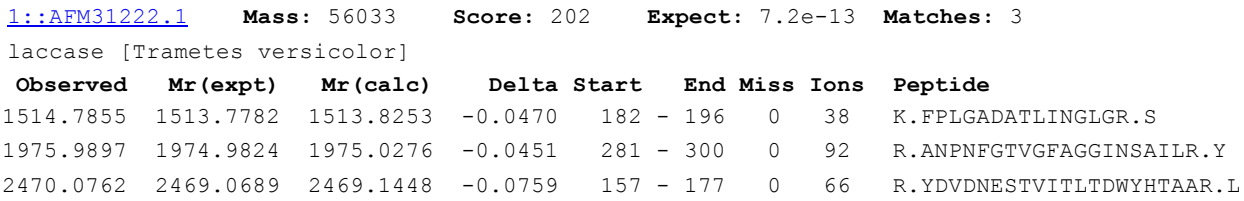

No match to: $731.4565,745.4716,751.3851,761.4732,804.2769,819.5120,832.3038,1243.6450,1474.6757,832.3132,834.3159$, $1703.7522,1742.7133,1948.0842,1991.9727,2021.9513,2043.0476,2070.0222,2087.9597,2101.9629,2150.1257,2199.0493$, $1243.6581,1474.6874,1514.7983,1515.7916,3214.6296,3247.3425,1703.7673,1705.7672,1974.0721,1976.0077,1991.9894$, $2059.9446,2070.0339,2087.9773,2150.1462,2158.0591,2199.0723,2470.0964,3214.6680,3247.3762$

\section{Search Parameters}

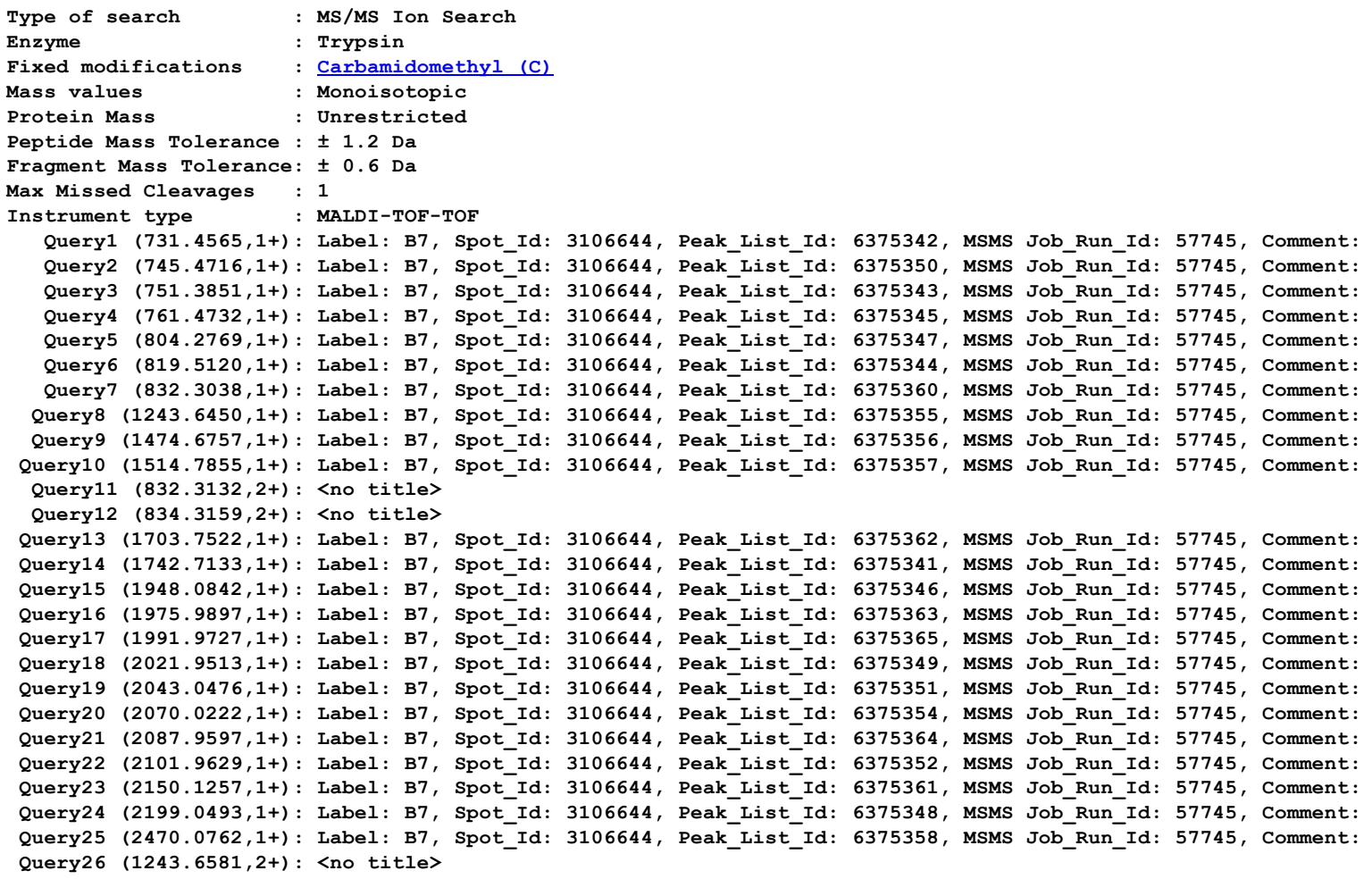


2/22/2017 Protein Summary Report (Project: ABSCIEX SERVICE, Spot Set: ABSCIEX SERVICEl080316, Label: B7, Spot Id: 3106644, Peak List Id: 6375246, MS...

Query27 (1474.6874,2+): <no title>

Query28 $(1514.7983,2+):<$ no title>

Query29 (1515.7916,2+): <no title

Query30 $(3214.6296,1+)$ : Label: B7, Spot Id: 3106644, Peak List Id: 6375359, MSMS Job Run Id: 57745, Comment:

Query31 $(3247.3425,1+)$ : Label: B7, Spot_Id: 3106644, Peak_List_Id: 6375353, MSMS Job_Run_Id: 57745, Comment:

Query32 (1703.7673,2+): <no title>

Query33 (1705.7672,2+): <no title

Query34 (1974.0721,2+): <no title>

Query35 (1976.0077,2+): <no title>

Query36 (1991.9894,2+): <no title>

Query37 (2059.9446,2+): <no title>

Query38 $(2070.0339,2+):<$ no title

Query39 (2087.9773,2+): <no title>

Query40 $(2150.1462,2+)$ : <no title>

Query41 (2158.0591,2+): <no title>

Query42 (2199.0723,2+): <no title>

Query43 (2470.0964,2+): <no title>

Query44 (3214.6680,2+): <no title>

Query45 $(3247.3762,2+)$ : <no title> 